\title{
Caractérisation de la structure démographique des ligneux dans les parcs agroforestiers du terroir de Dan Saga (Aguié, Niger)
}

\author{
Boubé MOROU ${ }^{1 *}$, Haoua OUNANI ${ }^{1}$, Abdoulaye AMADOU OUMANI ${ }^{1}$, \\ Abdoulaye DIOUF ${ }^{1}$, Chaïbou GUERO ${ }^{2}$ et Ali MAHAMANE ${ }^{3}$ \\ ${ }^{1}$ Université Dan Dicko Dankoulodo de Maradi, BP : 465 Maradi, Niger. \\ ${ }^{2}$ PASADEM, Maradi, BP : 473 Maradi, Niger. \\ ${ }^{3}$ Université de Diffa, BP : 78 Diffa, Niger. \\ *Auteur correspondant ; E-mail : boubem@yahoo.fr; BP : 465 Maradi, Niger; \\ Tél. : (00227) 96572215
}

\section{REMERCIEMENTS}

Les auteurs tiennent à remercier vivement le Projet d'Appui à la Sécurité Alimentaire et au Développement dans la Région de Maradi (PASADEM) pour son appui financier, à travers la convention signée avec l'UDDM de Maradi.

\section{RESUME}

La régénération naturelle assistée est une technique de préservation des ligneux dans les parcs agroforestiers pratiquée par les populations du terroir villageois de Dan Saga depuis plus de 20 ans. Cependant, son impact sur les caractéristiques structurales et écologiques reste à déterminer. Cette étude vise à caractériser les parcs agroforestiers du terroir villageois de Dan Saga et à déterminer la structure démographique des peuplements ligneux et des espèces ligneuses les plus dominantes et utilisées par les populations. Pour ce faire, un inventaire forestier a été réalisé dans 80 placettes de $2500 \mathrm{~m}^{2}(50 \mathrm{~m}$ x $50 \mathrm{~m})$ chacune. La régénération a été évaluée dans des placeaux de $25 \mathrm{~m}^{2}$. Les structures démographiques construites ont été ajustées au modèle de Weibull à 3 paramètres. Les résultats obtenus ont permis d'identifier trois parcs agroforestiers à savoir: le parc à Tamarindus indica et Grewia tenax, le parc à Annona senegalensis et Faidherbia ablida, et le parc à Albizia chevalerie et Cassia singuena. La structure en diamètre des peuplements ligneux et des espèces dominantes se caractérise par une densité importante d'individus dans les classes de petits diamètres. Ces données sont utiles pour une gestion durable des parcs agroforestiers.

(C) 2016 International Formulae Group. All rights reserved.

Mots clés : Parc agroforestier, peuplement ligneux, structure démographique, Dan Saga, Niger.

\section{Characterization of the woody stand demographic structure in the Dan Saga agroforestry parklands (Aguié, Niger)}

\section{ABSTRACT}

Tree assisted natural regeneration is a preserving technique of woody plants in the agroforestry parklands which is practiced by the population of Dan Saga village for over 20 years. Its impact on structural 
and ecological characteristics is not determined. This study aims at characterizing the agroforestry parklands of Dan Saga village, to determine the demographic structure of the woody stands. It is also aimed at determining the most dominant and used woody species by the populations. To do this, a forest census was conducted in 80 plots of $2500 \mathrm{~m}^{2}(50 \mathrm{~m} \mathrm{X} 50 \mathrm{~m})$ each. Tree natural regeneration was evaluated in smaller plots of $25 \mathrm{~m}^{2}$. The demographic structure built was adjusted to Weibull model with 3 parameters. Results revealed that three different agroforestry parklands were identified namely the Tamarindus indica and Grewia tenax one, the Annona senegalensis and Faidherbia ablida one, and the Albizia knighthood and Cassia singuena one. Structure in diameter of the tree stands and of the dominant species, is characterized by a high density of individual trees in the small diameter classes. These data are useful to plan a sustainable management of these parklands.

(C) 2016 International Formulae Group. All rights reserved.

Keywords: Agroforestry parkland, woody stands, demographic structure, Dan Saga, Niger.

\section{INTRODUCTION}

Depuis la fin des années 1960, le Niger est en proie à des sécheresses épisodiques marquées par le dépérissement des ligneux (Morou, 2010). Le terroir de Dan Saga dans le Département d'Aguié ne fait pas exception à la règle. En effet, à l'aridification s'ajoute une croissance démographique galopante ayant pour conséquence une extension du front agricole et une surexploitation des produits forestiers ligneux et non ligneux. Selon Ichaou (2000), chaque année, on assiste à un défrichement de 60000 ha en moyenne des formations contractées des plateaux du Niger. La zone sahélienne des pays membres du Comité Inter-Etats de Lutte contre la Sécheresse dans le Sahel (CILSS), a connu un recul annuel de $1,07 \%$ des formations forestières au cours de la période 2005-2010 (FAO, 2010). Cette dégradation se manifeste par des modifications de la composition floristique et de la structure de la végétation (Bakhoum, 2013). Cet état de fait se caractérise par la baisse de densité et de la diversité floristique des ressources ligneuses dont dépend fortement la population locale. La régression de la densité et de la qualité des espèces ligneuses dans la zone sahélienne a été signalée par Ganaba (2008) et Paré (2008) au Burkina Faso. Une solution à cette dégradation accélérée est la conservation des ressources forestières dans les parcs agroforestiers. Cette prise de conscience face à la préservation de la biodiversité s'est traduite par la mise en place des dispositions réglementaires pour protéger les terres forestières (Abdourhamane et al., 2013).

Conscientes de cette problématique, les populations de Dan Saga pratiquent depuis une vingtaine d'année la Régénération Naturelle Assistée (RNA) dans leurs champs. Selon des études menées dans les régions de Maradi et Zinder par Larwanou et Saadou (2011), on assiste à un reverdissement grâce aux efforts de réhabilitation effectués par les agriculteurs à travers la pratique de la RNA dans les parcs agroforestiers. Malheureusement, il manque encore des données sur la structure de la végétation dans les zones où la RNA est pratiquée permettant d'apprécier les effets de cette pratique sur les caractéristiques de la végétation. En effet, les données sur la structure et la diversité fournissent des éléments indicateurs permettant d'analyser les tendances d'évolution qualitative et quantitative de la végétation (Ouédraogo, 2006). Il importe alors de connaître les ressources forestières que renferment ces parcs agroforestiers pour un meilleur aménagement. Cette étude vise donc à évaluer les caractéristiques structurales et écologiques des ressources forestières dans le terroir de Dan Saga, vingt ans après l'adoption de la RNA. 


\section{MATERIEL ET METHODES}

\section{Site d'étude}

La présente étude a été menée dans le terroir villageois de Dan Saga (Département d'Aguié) localisé dans la région de Maradi au Centre-Ouest du Niger (Figure 1). Le climat est de type tropical sec avec une pluviométrie moyenne de $445,15 \mathrm{~mm}$ pour la période 1981 2010. La saison des pluies s'étend de mai à octobre. La température moyenne maximale varie de $30^{\circ} \mathrm{C}$ en janvier à $41^{\circ} \mathrm{C}$ en avril. Les conditions géomorphologiques permettent de dintinguer trois types de sols différenciés par les matériaux d'origine. C'est ainsi qu'on distingue les sols dunaires, les sols ferrugineux tropicaux et les sols des bas fonds. La végétation naturelle est constituée de steppe arbustive à arborée sur des dunes fixées. Les principales activités de la population d'Aguié sont l'agriculture et l'élevage. L'agriculture est essentiellement pluviale avec le mil, le sorgho et le niébé comme principales cultures. L'élevage qui constitue la seconde activité de la population se pratique sous trois formes, à savoir la transhumance (élevage extensif), l'élevage sédentaire traditionnel et l'élevage amélioré (embouche). C'est une zone relativement peuplée (environ 120 habitants au $\mathrm{Km}^{2}$ ).

\section{Echantillonnage et collecte des données}

Un échantillonnage par transect radiaire a été réalisé dans le cadre de cette étude. Ainsi, huit transects ont été installés suivant un gradient d'anthropisation à partir du village de Dan Saga. Sur chaque transect, dix placettes de $2500 \mathrm{~m}^{2}(50 \mathrm{~m} \quad \mathrm{x} \quad 50 \mathrm{~m})$ équidistantes de 300 ont été placés; soit 80 placettes au total. Les prémières placettes ont été installées à $300 \mathrm{~m}$ du village.

Pour la caractérisation de la végétation ligneuse, un inventaire forestier a été réalisé dans chaque placette. Les paramètres dendrométriques tels que le diamètre à $1 \mathrm{~m} 30$ (pour les arbres) et $20 \mathrm{~cm}$ au dessus du sol (pour les arbustes multicaules), la hauteur totale et les deux diamètres du houppier ont été mésurés. Les mesures ont concerné les individus au diamètre supérieur ou égal à 5 cm (Sambou, 2004; Ouédraogo, 2009). Les individus dont le diamètre est inférieur à $5 \mathrm{~cm}$ sont considérés comme faisant partie de la régénération (Mahamane et Saadou, 2008). La régénération a été évaluée dans cinq placeaux de $25 \mathrm{~m}^{2}(5 \mathrm{~m} \times 5 \mathrm{~m})$ repartis dans les quatre angles et au centre de la placette. Les données relatives aux facteurs stationnels à savoir les coordonnées de la placette, la texture du sol, la géomorphologie, le type d'utilisation de terre et le type de facteur de perturbation ont été également collectées à travers l'observation directe sur le terrain.

\section{Analyse des données \\ Paramètres dendrométriques}

Les paramètres dendrométriques ont été d'abord calculés par placette, puis pour chaque parc agroforestiers en calculant la moyenne des valeurs des placettes. Ces paramètres ont été calculés également pour chacune des quatre espèces les plus importantes et les plus utilisées du terroir c'est-à-dire ayant les plus forts indices de valeur d'importance et d'utilisation.

$$
\text { Il s'agit: }
$$

- Le taux de recouvrement $(R)$ en pourcentage (\%) obtenu par la formule suivante :

$$
\begin{aligned}
& \mathrm{R}=\frac{R m}{\mathrm{~s}} X 100 \\
& \text { avec } \mathrm{Rm}=\frac{\pi}{4} \sum_{i=1}^{n} d i^{2} \\
& R m=\text { recouvrement de l'ensemble des }
\end{aligned}
$$
individus de l'espèce de la placette $\left(\mathrm{m}^{2}\right) ; d i=$ diamètre moyen du houppier de l'individu $i$ (m) ; $\mathrm{s}=$ superficie de la placette $\left(\mathrm{m}^{2}\right)$.

- La densité moyenne en tiges $(N)$ exprimée en tiges par hectare (Tiges/ha) est déterminée par le nombre total de tiges dans chaque placette suivant la formule : $\mathrm{N}=\frac{n}{s}$ $n=$ nombre total de tiges inventoriées dans la placette et $s=$ superficie de la placette en hectare. 
- La densité moyenne en régénération $(N r)$ est exprimée en tiges par hectare (Tiges/ha) par la formule suivante : $\mathrm{Nr}=\frac{n r}{s}$ $n r=$ nombre total de tiges de diamètre inférieur à $5 \mathrm{~cm}$ recensées dans la placette et $\mathrm{s}$ $=$ superficie de la placette en hectare.

$$
\mathrm{D}_{\mathrm{g}}=\sqrt{\frac{1}{\mathrm{n}} \sum_{\mathrm{i}=1}^{\mathrm{n}} \mathrm{d}_{\mathrm{i}}^{2}}
$$

Le diamètre moyen $(\mathrm{Dg})$ exprimé en centimètre $(\mathrm{cm})$ est déterminé à l'échelle de chaque placette par la formule :

$n=$ nombre total de tiges rencontrées dans la placette et $d i=$ diamètre de la tige $\mathrm{i}(\mathrm{cm})$.

- La surface terrière globale $(G)$, exprimée en $\mathrm{m}^{2} / \mathrm{ha}$, est donnée par la formule :

$$
\mathrm{G}=\frac{\pi}{40000 \mathrm{~s}} \sum_{\mathrm{i}=1}^{\mathrm{n}} \mathrm{d}_{\mathrm{i}}^{2}
$$

$s=$ superficie de la placette en hectare et $d i=$ diamètre de la tige $\mathrm{i}(\mathrm{cm})$.

- La hauteur moyenne de Lorey $\left(H_{L}\right)$ exprimé en mètre $(\mathrm{m})$ est la hauteur moyenne des individus pondérés à leur surface terrière La formule est la suivante :

$$
H_{L}=\frac{\sum_{i=1}^{n} g_{i} h_{i}}{\sum_{i=1}^{n} g_{i}} \quad \text { avec } \quad g_{i}=\frac{\pi}{4} d_{i}^{2}, \text { Où }
$$

$g_{i}$ et $h_{i}$ sont respectivement la surface terrière et la hauteur totale de l'individu $i$.

Le test de Kruskal Walis a permis de faire le test de significativité des moyennes des paramètres dendrométriques calculés d'une part pour chaque parc et d'autre part pour chacune des quatre espèces choisies pour leurs importances écologiques et ethnobotaniques dans le terroir.

\section{Individualisation des parcs}

Pour la caractérisation des différents parcs, une Analyse Canonique de Correspondance (ACC) a été effectuée avec le logiciel PC-ORD 5.0 (McCune et Grace, 2002). Pour identifier les espèces qui contribuent significativement au maintien des parcs agroforestiers, les espèces en présence/absence dans les relevés, ont été testées par Indicator Species Analysis (ISA) (Dufrêne et Legendre, 1997) disponible sur $P C$-ORD 5 (McCune et Grace, 2002). Toutes les espèces ayant une probabilité (P) inférieure à 0,05 ont été retenues comme espèces caractéristiques du parc (Ouôba, 2006). Pour nommer le parc, il a été utilisé la combinaison de deux espèces, ayant les plus grandes valeurs indicatrices, les plus faibles valeurs de P (probabilité).

\section{Distributions en classe de diamètre des ligneux}

L'ensemble des individus de diamètre supérieur à $5 \mathrm{~cm}$ de toutes les espèces recensées ont été répartis par classes de diamètre d'amplitude $10 \mathrm{~cm}$. Par contre, les quatre espèces les plus importantes du terroir et les plus utilisées par la populations ont été répartis par classes de diamètre d'amplitude 5 cm. Aussi, les densités d'individus (en individus/ha) par classes de diamètre ont été déterminées.

Les différentes structures construites ont été ajustés au modèle de Weibull à 3 paramètres ( $a, b$ et $c$ ) à l'aide du logiciel Minitab 14. La fonction de densité de probabilité de la distribution de Weibull, se présente sous la forme de l'équation suivante (Rondeux, 1999) :

$$
f(x)=\frac{c}{b}\left(\frac{x-a}{b}\right)^{c-1} \exp \left[-\left(\frac{x-a}{b}\right)^{c}\right]
$$

Où $\mathrm{x}$ est la circonférence (diamètre ou hauteur) des arbres et $\mathrm{F}(\mathrm{x})$ sa valeur de densité de probabilité ; $a$ est le paramètre de position $(5 \mathrm{~cm}) ; b$ est le paramètre d'échelle ou de taille ; $c$ est le paramètre de forme lié à la structure en diamètre (circonférence) ou hauteur considérée.

Une analyse log-linéaire a été réalisée avec le logiciel R 2.15.3 pour comparer dans chaque cas, la distribution en diamètre observée à la distribution théorique de Weibull avec estimation des paramètres. L'hypothèse de l'ajustement entre les deux distributions (théorique et observée) a été acceptée si la valeur de la probabilité du test est supérieure à 0,05 . 


\section{RESULTATS}

Individualisation des pares par Analyse Canoniques de Correspondances (ACC)

Il ressort de l'ordination par l'ACC de la matrice des relevés-espèces ( 80 relevés et 37 espèces) de l'ensemble de la végétation en fonction des variables environnementales (occupation et texture), trois parcs agroforestiers, notés G1, G2 et G3 (Figure 2). Ils sont corrélés aux deux premiers axes de l'ACC qui résument $58,8 \%$ de la variance totale. Ces axes sont également corrélés aux variables texture et occupation du sol. L'axe 1 est mieux corrélé avec la variable "texture" $(r=0,62)$ et au parc $\mathrm{G} 1$, exprime ainsi un gradient de texture du sol. Quant à l'axe 2, il exprime un gradient d'occupation du sol car mieux corrélé mais négativement à la variable "occupation" $(r=-0,39)$ et au parc G3. Le parc G1 correspond ainsi à un peuplement ligneux constitué des espèces qu'on retrouve sur des types de sol bien déterminés et G3 à un peuplement ligneux dominé par les espèces retrouvées dans tous les types d'occupation du sol. Quant au parc G2 elle correspond aux peuplements ligneux influencés à la fois par la texture et l'occupation du sol.

L'utilisation de la combinaison des deux premières espèces ayant les plus grandes valeurs indicatrices et les plus faibles valeurs de probabilité permet de nommer les trois (3) types de parc.

Le parc à Tamarindus indica et Grewia tenax (G1) se localise au niveau des sols sablo-argileux communément appelés «Gueza» et comporte 15 relevés dont 2 effectués dans les jachères. La présence des termitières est à notifier dans les relevés de ce parc. Celui à Annona senegalensis et Faidherbia albida (G2) se localise au niveau des sols sablo-argileux et sols sablo-limoneux (dunaires) et comporte 44 relevés effectués dans les champs. Le parc à Albizia chevalerie et Cassia singuena (G3) quant à lui se localise surtout au niveau des sols sablo-limoneux (dunaires) communément appelés «Jigawa» et comporte 21 relevés dont 5 effectués dans les jachères.
Outre les deux espèces caractéristiques utilisées dans chaque cas, plusieurs espèces importantes accompagnent les espèces principales dans chaque parc. Ainsi, pour le parc G1, il s'agit des espèces comme Terminalia avicennioides, Calotropis procera et Maerua crassifolia. Pour le parc G2 on note la présence des espèces comme Balanites aegyptiaca, Piliostigma reticulatum et Hyphaene thebaica. Quant au parc G3, ce sont des espèces telles que Dichrostachys cirenea, Stereospermum kunthianum et Boscia senegalensis qui accompagnent les espèces principales.

\section{Indices de diversité}

L'analyse du Tableau 1 montre que la diversité est faible dans le terroir avec des indices de Shannon faibles. Pour l'ensemble du terroir, l'indice de diversité de Shannon $\left(\mathrm{H}^{\prime}\right)$ est de 2,2 bits et il varie de 1,79 (G1) à 2,16 (G2). Quant à l'équitabilité de Pielou (E) elle est globalement moyenne au niveau global et dans les différents parcs.

La richesse spécifique varie de 20 (G1) à 31 (G2) espèces dans les différents parcs. Ce qui dénote une certaine différence de cortège floristique de ces parcs.

\section{Caractéristiques dendrométriques}

Il ressort de l'analyse globale du Tableau 2 qu'il existe une différence significative au seuil de $5 \%(\mathrm{P}<0,05)$ entre les différents parcs pour la densité de régénération $(\mathrm{Nr})$ et le diamètre moyen de la tige $(\mathrm{Dg})$ tandis que pour les autres paramètres à savoir la surface terrière $(\mathrm{G})$, la hauteur de Laurey $\left(\mathrm{H}_{\mathrm{L}}\right)$, le taux de recouvrement $(\mathrm{R})$ et la densité des adultes (Na) le test de Kruskall Wallis n'a relevé aucune différence significative au seuil de 5\% $\quad(\mathrm{P}>0,05)$. Néanmoins, pour l'ensemble des paramètres, les plus grandes valeurs sont observées pour G2 $\left(\mathrm{D}=21,7 \pm 1,19, \quad \mathrm{H}_{\mathrm{L}}=7,25 \pm 0,33, \quad \mathrm{G}=\right.$ $0,06 \pm 0,01$ et $R=14,18 \pm 1,29)$. La densité varie de 5,6 pieds/ha pour G1 à 7,09 pieds/ha pour G2. Quant à la densité de régénération, elle 
varie de 282 pieds/ha pour G2 à 462,5 pieds/ha pour G3.

\section{Structure en diamètre}

Les structures en diamètre des parcs ont été réalisées avec surimposition de la distribution de Weibull (Figure 3). Les résultats de l'analyse log-linéaire montrent que les distributions observées des différents parcs s'ajustent globalement avec les distributions de Weibull (P>0,05). Pour l'ensemble des parcs, la structure en diamètre des individus ligneux est caractéristique d'une distribution en « J renversé » avec le paramètre de forme (c) de valeur comprise entre 1 et 3,6 pour les parcs G1 et G2 caractéristiques des peuplements monospécifiques avec prédominance d'individus jeunes ou de petits diamètres et inférieure à 1 pour le parc G3 caractéristique des peuplements multispécifiques ou inéquiennnes. On observe une absence de la classe de diamètre comprise entre 55 et $75 \mathrm{~cm}$ pour le parc G1 et une quasi absence des individus de diamètre supérieur à $55 \mathrm{~cm}$ dans le parc G2. Quant au parc G3, on observe une absence des classes de diamètre comprises entre 35 et $45 \mathrm{~cm}$ et une quasi absence des individus de diamètre supérieur à $45 \mathrm{~cm}$.

\section{Importance écologique des espèces dans le terroir}

Les espèces qui ont les plus fortes valeurs d'Indice de Valeur d'Importance (IVI) sont Combretum glutinosum (90), Faidherbia albida (60), Piliotigma reticulatum (38), Gueira senegalensis (16) et Annona senegalensis (16). Ces espèces marquent la physionomie de la végétation du terroir de Dan Saga. En effet, leur IVI représente $73,33 \%$ de l'IVI total.

\section{Caractéristiques dendrométriques des espèces importantes}

Le Tableau 3 donne une comparaison des moyennes des paramètres dendrométriques des 4 espèces les plus importantes et les plus exploitées du terroir. Il ressort de l'analyse globale de ce tableau qu'il existe une différence très significative $(\mathrm{P} \leq 0,001)$ entre les différentes espèces pour la surface terrière $(G)$, la hauteur de Lorey $\left(\mathrm{H}_{\mathrm{L}}\right)$, le diamètre $(\mathrm{Dg})$ et le taux de recouvrement (R). Ainsi, le plus faible diamètre est observé pour $G$. senegalensis $(7,4)$ tandis que pour le reste des paramètres leurs plus faibles valeurs sont observées pour A. senegalensis $\left(\mathrm{H}_{\mathrm{L}}=\right.$ $2,14 \pm 0,14, \mathrm{G}=0,03 \pm 005$ et $\mathrm{R}=0,45 \pm 0,1$ ) et les plus grandes valeurs pour l'ensemble des paramètres sont observées pour $C$. glutinosum $\left(\mathrm{D}=19,16 \pm 1,05, \mathrm{H}_{\mathrm{L}}=6,77 \pm 0,31, \mathrm{G}=1,52 \pm 0,2\right.$ et $\mathrm{R}=6,38 \pm 01)$. La densité varie de 6 pieds/ha pour A. senegalensis à 13 pieds/ha pour $C$. glutinosum. Quant à la densité de régénération, elle varie de 115 pieds/ha pour A. senegalensis à 593 pieds/ha pour $G$. senegalensis.

\section{Structures en diamètre}

Les structures en diamètre des 4 espèces ont été réalisées avec surimposition de la distribution de Weibull (Figure 4). Les résultats de l'analyse log-linéaire montrent que les distributions observées des différentes espèces s'ajustent globalement avec les distributions de Weibull respectives ( $\mathrm{P}$ > 0,05). Pour l'ensemble des 4 espèces, la structure en diamètre des individus ligneux est caractéristique d'une distribution en « J renversé » avec le paramètre de forme (c) de valeur comprise entre 1 et 3,6 caractéristiques des peuplements monospécifiques avec dominance d'individus jeunes ou de petits diamètres. Pour l'ensemble des espèces, on remarque une dominance des individus de diamètres compris entre $5 \mathrm{~cm}$ et $10 \mathrm{~cm}$ comme l'indique les structures en diamètre.

$\mathrm{Cg}$ : Combretum glutinosum, Pr: Piliostigma reticulatum, Gs: Guiera senegalensis et As: Annona senegalensis. 

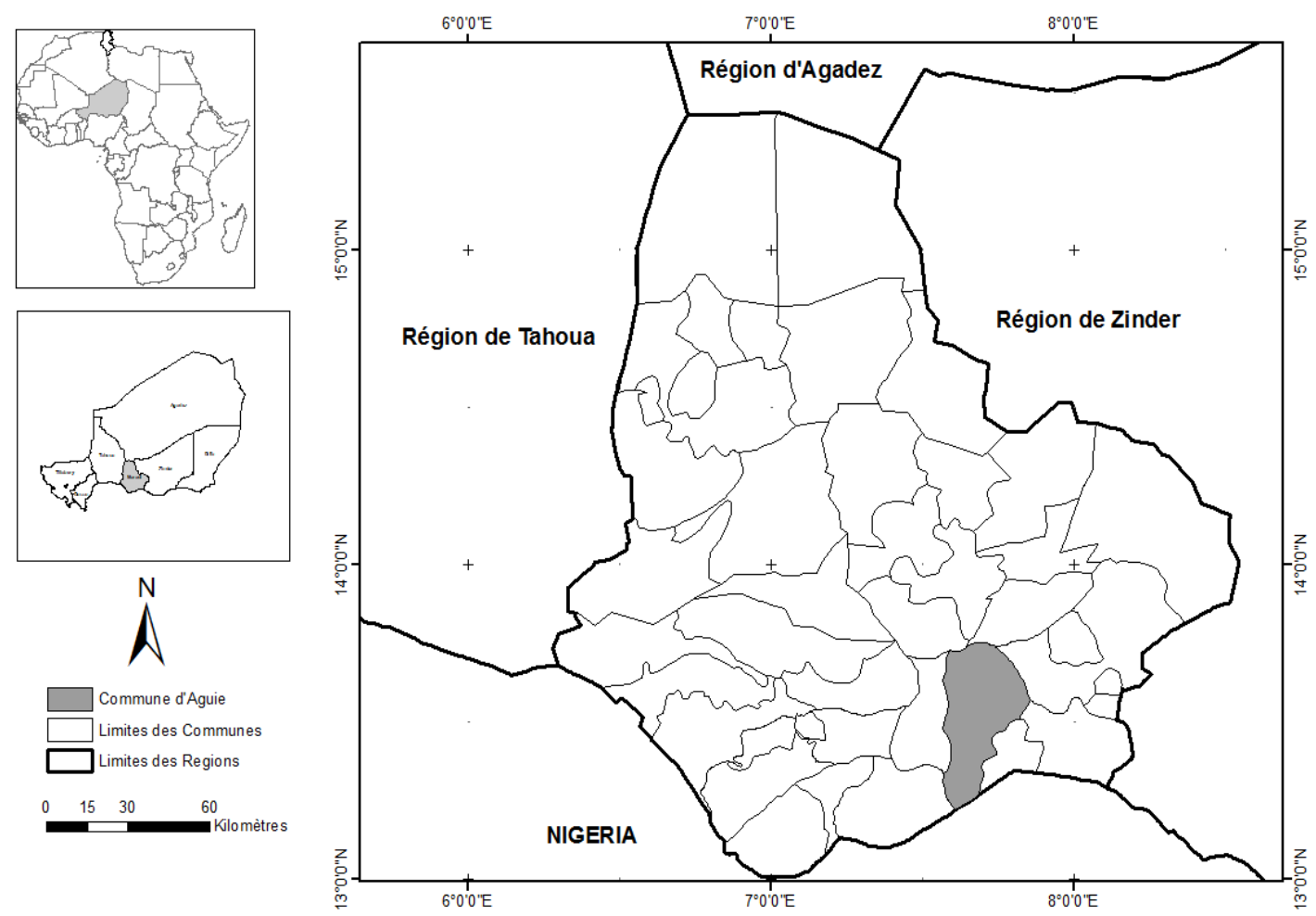
Source: Département des Sciences du sol et de Télédétection, Faculté d'Agronomie et des Sciences de l'Environnement, Université de Maradi, Niger
Auteur: Abdoulaye DIOUF

Figure 1 : Carte de la région de Maradi avec la localisation du département d'Aguié.

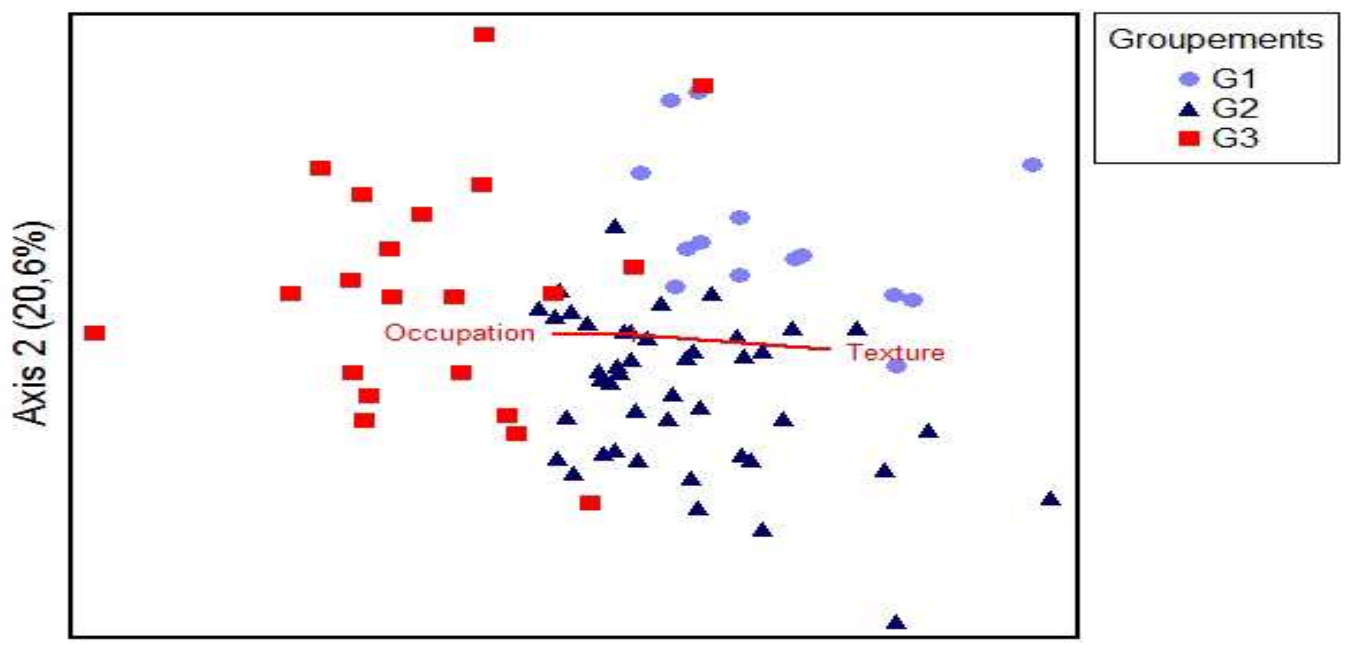

Axis $1(38,2 \%)$

Figure 2 : Analyse Canoniques de Correspondances des parcs agroforestiers. 
B. MOROU et al. / Int. J. Biol. Chem. Sci. 10(3): 1295-1311, 2016
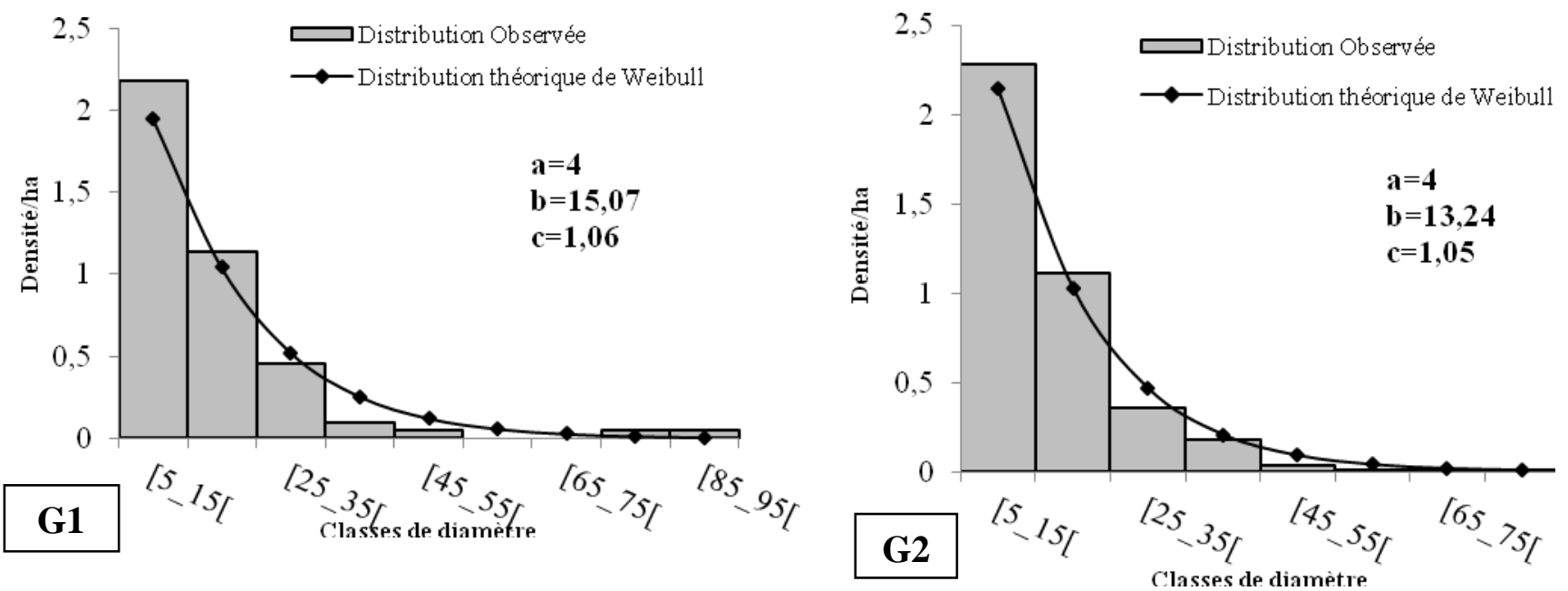
B. MOROU et al. / Int. J. Biol. Chem. Sci. 10(3): 1295-1311, 2016

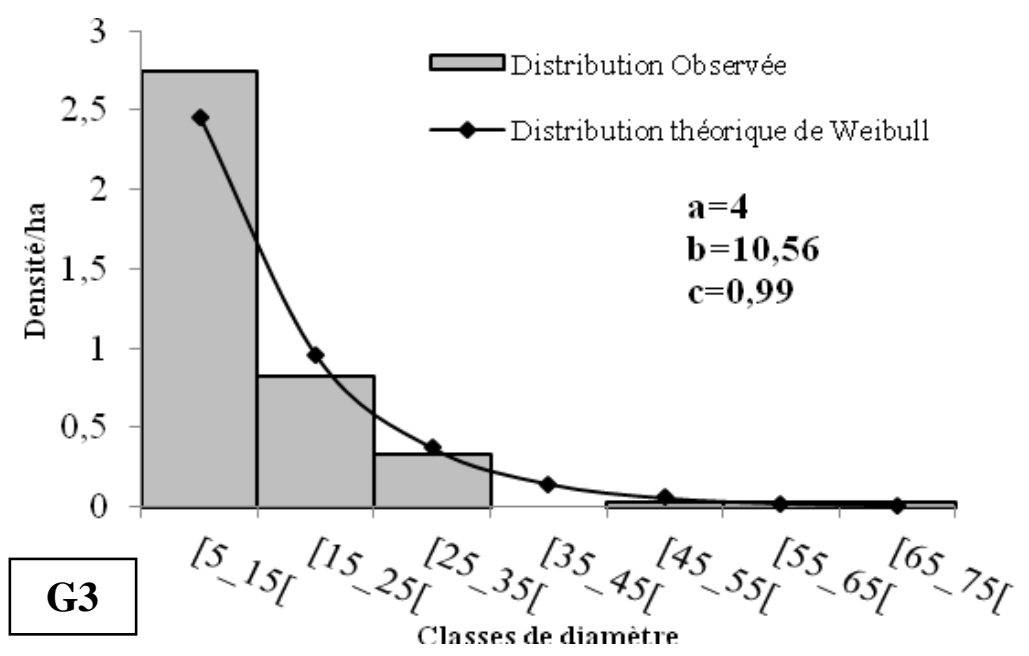

Figure 3 : Structure des parcs agroforestiers par classes de diamètre. 

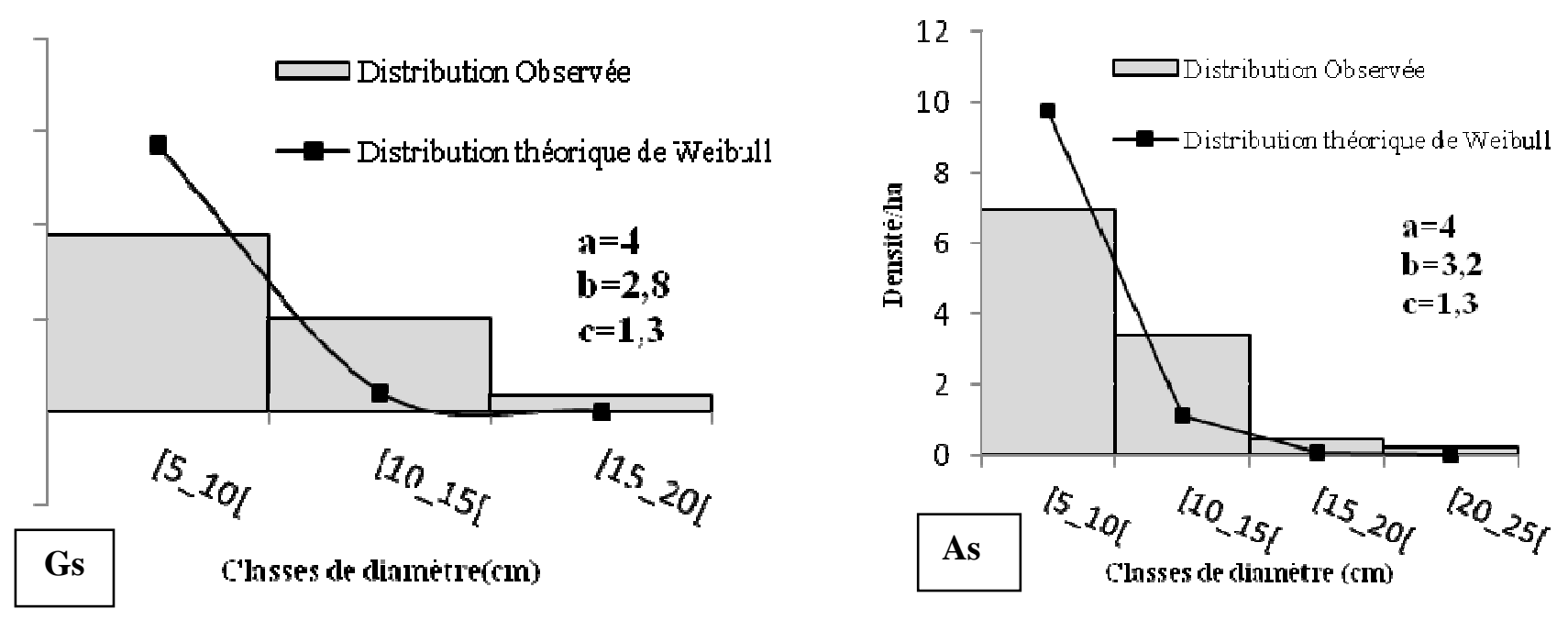

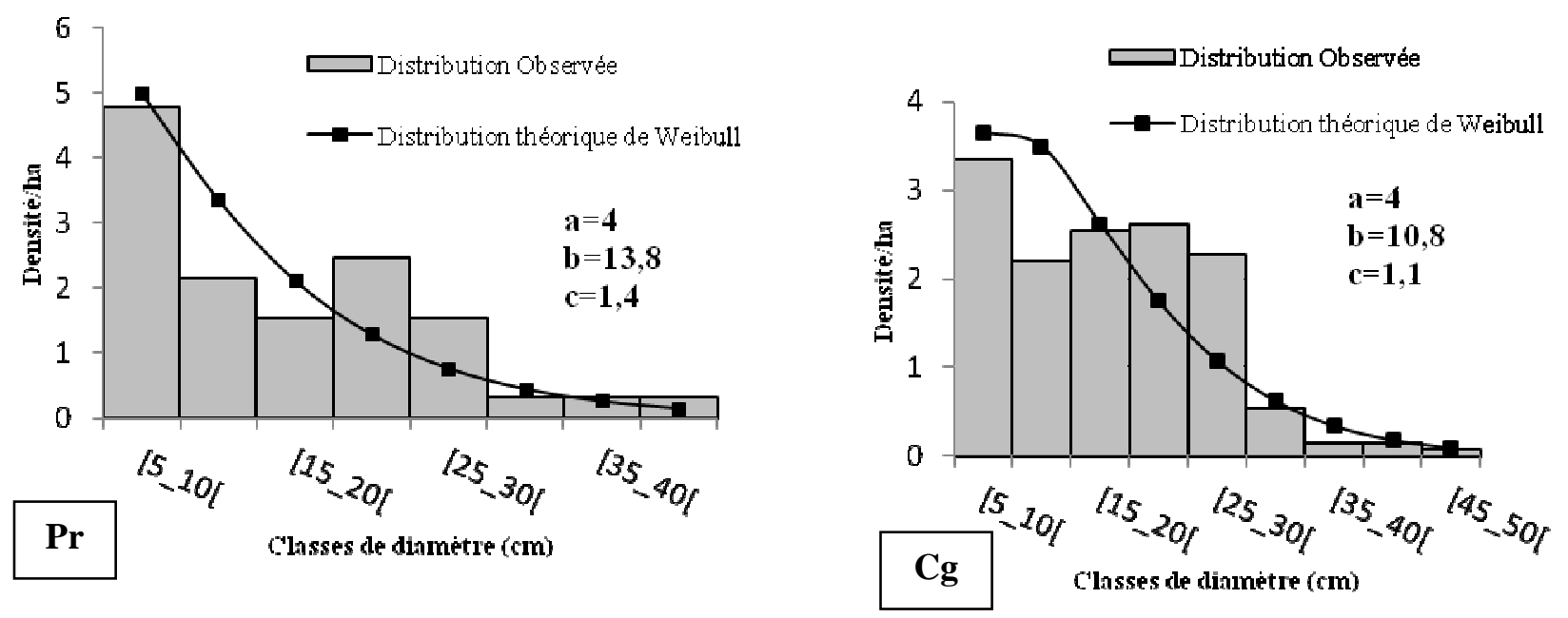

Figure 4 : Structure par classes de diamètre des quatre espèces les plus importantes et les plus utilisées du terroir. 
Tableau 1 : Diversité du terroir.

\begin{tabular}{lcccc}
\hline & Globale & G1 & G2 & G3 \\
\hline Shannon (H en bits) & 2,2 & 1,79 & 2,16 & 1,9 \\
Richesse spécifique (R) & 37 & 20 & 31 & 27 \\
Diversité maximale (Hmax) & 3,6 & 3 & 3,4 & 3,3 \\
Equitabilité (E) & 0,63 & 0,6 & 0,63 & 0,58 \\
\hline
\end{tabular}

Tableau 2 : Comparaison des moyennes des paramètres dendrométriques des parcs agroforestiers du terroir.

\begin{tabular}{|c|c|c|c|c|c|c|c|}
\hline & \multicolumn{2}{|c|}{ G1 } & \multicolumn{2}{|c|}{ G2 } & \multicolumn{2}{|c|}{ G3 } & \multirow{2}{*}{$\mathbf{P}$} \\
\hline & m & $\sigma$ & $\mathbf{M}$ & $\sigma$ & $\mathbf{M}$ & $\boldsymbol{\sigma}$ & \\
\hline $\mathrm{D}(\mathrm{cm})$ & $21,56^{\mathrm{a}}$ & 3,32 & $21,7^{\mathrm{a}}$ & 1,19 & $16,5^{b}$ & 1,37 & 0,02 \\
\hline $\mathrm{H}_{\mathrm{L}}(\mathrm{m})$ & $7,03^{\mathrm{a}}$ & 0,8 & $7,25^{\mathrm{a}}$ & 0,33 & $7,14^{\mathrm{a}}$ & 0,73 & 0,74 \\
\hline $\mathrm{G}\left(\mathrm{m}^{2} / \mathrm{ha}\right)$ & $0,06^{\mathrm{a}}$ & 0,02 & $0,06^{\mathrm{a}}$ & 0,01 & $0,04^{\mathrm{a}}$ & 0,01 & 0,10 \\
\hline $\mathrm{R}(\%)$ & $12,31^{\mathrm{a}}$ & 2,65 & $14,18^{\mathrm{a}}$ & 1,29 & $12,44^{\mathrm{a}}$ & 1,78 & 0,60 \\
\hline $\mathrm{Na}$ (pieds/ha) & $5,6^{\mathrm{a}}$ & 0,85 & $7,09^{\mathrm{a}}$ & 0,57 & $6,52^{\mathrm{a}}$ & 0,71 & 0,44 \\
\hline $\mathrm{Nr}$ (pieds/ha) & $372,6^{\mathrm{a}}$ & 34,6 & $282^{\mathrm{b}}$ & 20,3 & $462,5^{\mathrm{c}}$ & 25,3 & 0,00 \\
\hline
\end{tabular}

G1 : parc 1; G2 : parc 2; G3 : parc 3 ; P : Probabilité ; D : Diamètre; $\mathrm{H}_{\mathrm{L}}$ : Hauteur de Lorey ; G : surface terrière; $\mathrm{R}$ : recouvrement; $\mathrm{Nr}$ : Densité de régénérations ; $\mathrm{Na}$ : densité des adultes ; $\mathrm{m}$ : moyenne, $\sigma$ : écart type Sur une même ligne, les valeurs accompagnées de lettres différentes sont significativement différentes.

Tableau 3: Comparaison des moyennes des paramètres dendrométriques des 4 espèces les plus importantes et les plus utilisées.

\begin{tabular}{|c|c|c|c|c|c|c|c|c|c|}
\hline & \multicolumn{2}{|c|}{ As } & \multicolumn{2}{|c|}{$\mathrm{Cg}$} & \multicolumn{2}{|c|}{ Gs } & \multicolumn{2}{|c|}{ Pr } & \multirow[t]{2}{*}{$\mathbf{p}$} \\
\hline & $\mathbf{M}$ & $\boldsymbol{\sigma}$ & $\mathbf{m}$ & $\boldsymbol{\sigma}$ & $\mathbf{m}$ & $\boldsymbol{\sigma}$ & $\mathbf{m}$ & $\boldsymbol{\sigma}$ & \\
\hline $\mathrm{D}(\mathrm{cm})$ & $11,92^{\mathbf{a}}$ & 2,3 & $19,16^{\mathbf{b}}$ & 1,05 & $7,4^{\mathrm{c}}$ & 0,7 & $17,5^{\mathrm{d}}$ & 1,7 & 0,000 \\
\hline $\mathrm{H}_{\mathrm{L}}(\mathrm{m})$ & $2,14^{\mathrm{a}}$ & 0,14 & $6,77^{\mathbf{b}}$ & 0,31 & $3,43^{\mathrm{c}}$ & 0,22 & $4,41^{\mathrm{d}}$ & 0,27 & 0,000 \\
\hline $\mathrm{R}(\%)$ & $0,45^{\mathbf{a}}$ & 0,1 & $6,38^{\mathbf{b}}$ & 0,61 & $2,12^{\mathrm{c}}$ & 0,64 & $4,35^{\mathbf{d}}$ & 0,77 & 0,000 \\
\hline $\mathrm{G}\left(\mathrm{m}^{2} / \mathrm{ha}\right)$ & $0,03^{\mathrm{a}}$ & 0,005 & $1,52^{\mathbf{b}}$ & 0,2 & $0,1^{\mathrm{c}}$ & 0,04 & $0,44^{\mathrm{d}}$ & 0,08 & 0,000 \\
\hline $\mathrm{Nr}$ (pieds/ha) & $115,1^{\mathrm{a}}$ & 14,5 & $234,8^{\mathrm{b}}$ & 30,3 & $593,3^{\mathrm{c}}$ & 78,2 & $164,5^{\mathrm{d}}$ & 18,4 & 0,000 \\
\hline $\mathrm{Na}$ (pieds/ha) & $6,18^{\mathrm{a}}$ & 0,77 & $12,67^{\mathrm{b}}$ & 1,47 & $9,05^{\mathrm{c}}$ & 1,73 & $8,75^{\mathrm{d}}$ & 1,47 & 0,001 \\
\hline
\end{tabular}




\section{DISCUSSION}

\section{Composition floristique}

La richesse spécifique du terroir est de 37 espèces et varie de 20 à 31 . Ces résultats sont similaires à ceux de Amina (2004) qui a trouvé 22 espèces dans le terroir de Dan Saga et ceux de Dan Guimbo (2011) qui a trouvé 35 espèces dans le parc à Vitellaria paradoxa et 22 espèces dans celui à Neocarya macrophylla dans le Sud-Ouest du Niger. Une légère différence de composition floristique a été observée dans les différents groupements. Ce qui pourrait être expliqué par la différence de substrat sur lequel ces groupements évoluent. En effet, la répartition des espèces ligneuses est conditionnée par les facteurs climatiques et édaphiques (Ndong et al., 2015). Selon Baggnian et al. (2014), la composition et la structure de la végétation ligneuse varient considérablement d'une localité à l'autre en fonction des facteurs environnementaux et des perturbations anthropiques.

Les espèces ligneuses les plus fréquentes de façon globale et dans les différents parcs sont $C$. glutinosum avec le plus fort IVI suivi de $F$. albiba, $P$. reticulatum, $G$. senegalensis et $A$. senegalensis en adultes et jeunes individus. Ces espèces marquent la physionomie du terroir. Ceci peut être expliqué par l'assistance des agriculteurs dont bénéficient les espèces ligneuses en général dans les champs et particulièrement les espèces ayant un impact positif sur l'amélioration du niveau de fertilité des sols comme $F$. albida, $G$. senegalensis et G. glutinosum ce qui caractérise un certain degré de fertilité des sols dans le terroir. Matsallabi (2008) a trouvé que sous les pieds de G. senegalensis, le rendement de mil est de $600 \mathrm{~kg} / \mathrm{ha}$ alors qu'en absence de Guiera senegalensis, il est de $300 \mathrm{~kg} / \mathrm{ha}$. Ainsi, le rendement en grain est environ 2 fois plus important dans une parcelle couverte de la touffe de $G$. senegalensis que dans une parcelle non couverte de la touffe de $G$. senegalensis. Abdourahamane (2006) a montré que le rendement à l'hectare évolue de $65 \mathrm{~kg} / \mathrm{ha}$ au niveau des parcelles sans arbre à $122 \mathrm{~kg} / \mathrm{ha}$ avec dominance d'autres espèces et $240 \mathrm{~kg} / \mathrm{ha}$ pour les parcelles à dominance de Faidherbia albida.

\section{Caractéristiques des parcs agroforestiers.}

Les caractéristiques communes à ces différents parcs sont le faible taux d'individus dans les classes de circonférences moyennes, ce qui peut être attribué à une coupe régulière et précoce des individus due aux activités anthropiques dans la zone. La distribution des différentes classes de circonférence montre que les faibles classes de circonférence sont bien représentées. Il s'agit généralement de peuplements dotés d'une régénération rapide. Les peuplements ligneux sont à physionomie dominée par les Combrétacées. Les faibles valeurs de l'indice de Shannon observées dans les deux parcs (G1 et G3) témoignent des phénomènes de dominance d'une espèce ou d'un groupe d'espèces à savoir $C$. glutinosum et $F$. albida. En effet, selon Morou (2010), l'indice de Shannon a des valeurs fortes pour des espèces avec des recouvrements de même importance et il prend des valeurs faibles lorsque quelques espèces ont de forts recouvrements. Les valeurs de l'indice d'équitabilité de Pielou observées confirment cette tendance.

\section{Caractéristiques structurales}

Les parcs de peuplement étudiés ont une densité des pieds adultes (ayant un diamètre de la tige supérieur ou égal à $5 \mathrm{~cm}$ ) comprise entre 5,6 et 7,09 tiges/ha avec une moyenne de 25,8 tiges/ha pour le peuplement global. Ces densités sont sensiblement les mêmes pour tous les parcs et sont faibles (pas de différence significative au seuil de 5\%). La faible densité des parcs pourrait être expliquée par la dominance des grands arbres 
caractérisés par une exploitation abusive et dont la reconstitution nécessite une longue durée sans nouvelles perturbations eut égard aux conditions écologiques. Cette tendance a été notée par Douma (2009) dans la réserve totale de faune de Tamou à l'Ouest du Niger.

Toutes les structures en diamètre obtenues pour les trois parcs identifiés sont ajustées à la distribution de Weibull et sont en "J renversé" avec une prédominance des individus de diamètre compris entre $5 \mathrm{~cm}$ et $15 \mathrm{~cm}$. Mais il est plutôt très rare de rencontrer des individus de diamètre supérieur à $50 \mathrm{~cm}$ dans le terroir, ce qui témoigne du repeuplement de cette formation où les individus les plus gros étaient certainement exploités de façon incontrôlée pour les besoins de la famille avant la prise de conscience effective de l'importante des arbres dans les champs. Selon Morou (2010), l'abondance des individus de classes de faible diamètre assurent l'avenir de la formation naturelle tandis que les individus de gros arbres résultent de la sélection naturelle et sont en fait les semenciers qui assurent la pérennité du peuplement. Une telle distribution est typique des populations stables, susceptibles de se renouveler par la régénération naturelle (Mbayngone et al., 2008). Cependant, Sambou (2004) ayant fait le même constat dans des forêts classées au Sénégal, a conclu qu'une analyse globale de la structure de la végétation cache un processus de dégradation qui affecte les populations de certaines espèces à fort potentiel socioéconomique.

Les taux de recouvrements, respectivement pour G1, G2 et G3 $(12,31 \%$, $14,18 \%$ et $12,44 \%$ ) peuvent s'expliquer par la présence des individus à grandes cimes dans ces parcs tels que Sclerocarya birrea, Tamarindus indica et $F$. albida. Ces taux de recouvrement pourraient traduire une faible productivité de ruissellement du terroir. Ces résultats sont élevés par rapport à ceux trouvés par Boubacar et al., (2013) sur les glacis $(3,01 \%)$ dans la Commune rurale de Simiri à l'Ouest du Niger. Cela peut s'expliquer par la faible densité en tiges dans ces agrosystèmes à cause de la texture sableuse fine et la faible teneur en matière organique des sols de ces agrosystèmes.

\section{Comportement des quatre espèces importantes}

Sur le plan structural, la densité des arbres est de 25,8 pieds/ha. La densité des espèces comme $C$. glutinosum, $P$. reticulatum, $G$. senegalensis et $A$. senegalensis varie de 6 à 12pieds/ha. Ces valeurs de densité peuvent s'expliquer par un fort degré de pression anthropique exercée sur ces espèces. Cette tendance est confirmée par les fortes régénérations observées pour chacune des espèces. Cette forte régénération pourrait témoigner de la capacité des espèces à utiliser des méthodes de multiplication végétative pour coloniser le milieu. C'est le cas de $G$. senagalensis qui a la plus forte densité de régénération. Ces résultats sont similaires à ceux de Abdourhamane et al., (2013) qui ont trouvé une abondance de G. senagalensis dans la forêt classée de Dan Kada Dodo Dan Gado dans le Centre-Sud du Niger.

\section{Structures en diamètre}

De façon générale, une diminution progressive du nombre d'individus lorsque la classe de diamètre augmente a été observée chez toutes les quatre espèces. Cette abondance de jeunes sujets peut être considérée aussi comme un indice de repeuplement de ces espèces dans le système agroforestier de la zone témoignant ainsi, de la capacité des populations actuelles à protéger les jeunes plants contre les pressions (piétements, broutages, mauvaise pratique d'exploitation). Elle témoigne également des phases vécues par ces espèces c'est-à-dire l'intérêt que leur accordaient dans le passé les 
exploitants dans les unités de gestion et les sécheresses récurrentes qui ont sévi dans la zone il y a une quinzaine d'années. Ces résultats sont contraires à ceux de Douma (2009) qui a trouvé dans la réserve totale de faune de Tamou à l'Ouest du Niger une structure en $« \mathbf{J} »$ pour Vitellaria paradoxa caractérisée par une répartition croissante des individus des petites classes de diamètre vers les grandes classes et une structure en « $\mathrm{U}$ » pour Adansonia digitata, et Parkia biglobosa caractérisée par une abondance des individus de gros diamètre et des petits diamètre et une absence de recrutement des individus dans les classes intermédiaires. Ceci pourrait être expliqué par la forte pression anthropique exercée sur cette réserve en général et sur ces espèces en particulier notamment par la pression de ramassage des fruits qui a déjà été signalé par Douma (2010) dans l'Ouest du Niger.

\section{Capacité de régénération des espèces}

On observe un fort taux de régénération pour l'ensemble des quatre espèces. Elle varie de 115 pour A. senegalensis à 593 pieds/ha pour $G$. senegalensis. La forte régénération de ces espèces pourrait être expliquée aussi par l'efficacité du mode de gestion et des outils de gestion utilisés par la population. En effet, la population de Dan Saga pratique plus la coupe au ras du sol pour favoriser l'émergence des rejets.

Le recouvrement basal exprimé en $\mathrm{m}^{2} / \mathrm{ha}$ est plus élevé pour $C$. glutinosum $(1,52)$, ce qui témoigne que les arbres de cette espèce s'adaptent mieux aux conditions écologiques du milieu et que ses rejets peuvent avoir une meilleure résistance vis-àvis des facteurs écologiques contrairement à ceux de $P$. reticultum $(0,44)$, A. senegalensis $(0,03)$ et $G$. senegalensis $(0,1)$. Ces faibles valeurs de surface terrières pourraient être expliquées par la pression anthropique exercée sur ces espèces.

\section{Conclusion}

Cette étude a permis de discriminer trois types de parcs agroforestiers dans le terroir villageois de Dan Saga: le parc à Tamarindus indica et Grewia tenax, le parc à Annona. senegalensis et Faidherbia. albida et celui à Albizia chevalieri et Cassia singuena. Ils présentent une diversité spécifique et des paramètres écologiques variables. La diversité floristique, la densité, la surface terrière et la structure démographique des peuplements ligneux sont le reflet du choix des espèces utiles et du mode de gestion des champs par les agriculteurs. Ces derniers modifient en effet la structure naturelle des peuplements ligneux par leurs pratiques culturales. La forte régénération des espèces pourrait être expliquée par l'efficacité du mode de gestion et des outils de gestion utilisés par la population.

\section{CONFLIT D'INTERETS}

Les auteurs de ce manuscrit déclarent qu'il n'y a aucun conflit d'intérêt entre eux.

\section{CONTRIBUTIONS DES AUTEURS}

Dans la réalisation de la présente étude, $\mathrm{BM}$ et $\mathrm{HO}$ ont élaboré le protocole de recherche, collecté et traité les données et aussi rédigé le manuscrit. $\mathrm{AAO}$ et $\mathrm{AD}$ ont participé à la relecture du document; $\mathrm{CG}$ et AM ont apporté des conseils dans la collecte et le traitement des données.

\section{REFERENCES}

Abdourhamane H, Morou B, Rabio H, Mahamane A. 2013. Caractéristiques floristiques, diversité et structure de la végétation ligneuse dans le Centre-Sud du Niger : cas du complexe des forêts classées de Dan kada Dodo-Dan Gado (région de Maradi, Niger). Int. J. Biol. Chem. Sci., 7(3): 1048-1068. DOI: http://dx.doi.org/10.4314/ijbcs.v7i3.13. 
Abdourahamane A. 2006. Evaluation de la régénération naturelle des ligneux dans les champs de la zone sud du département d'Aguié : cas des terroirs de Sadja Mandja et Dan Gamdji, mémoire pour l'obtention du diplôme d'ingénieur des techniques agricoles, FA, $70 \mathrm{p}$.

Amina M. 2004. Etude technique et de faisabilité et organisationnelle d'un marché de bois issus de la régénération naturelle dans la grappe de Dan Saga. Mémoire d'ingénieur de Techniques Agricoles, Faculté d'Agronomie, Université Abdou Moumouni de Niamey, p.76.

Baggnian I, Adam T, Adamou MM, Chaibou I, Mahamane A. 2014. Structure et dynamique de la végétation ligneuse juvénile issue de la régénération naturelle assistée (RNA) dans le CentreSud du Niger. Int. J. Biol. Chem. Sci., 8(2): 649-665. DOI : http://dx.doi.org/10.4314/ijbcs.v8i2.22.

Bakhoum A. 2013. Dynamique des ressources fourragères: indicateur de résilience des parcours communautaires de Téssékéré au Ferlo (Nord-Sénégal). Thèse de doctorat unique en en Biologie, Productions et Pathologies Animales, Option Ecologie pastorale, FST-UCAD, p.115.

Boubacar MM, Inoussa MM, Ambouta JMK, Mahamane A, Jorgen AA, Harissou Y, Rabiou H. 2013. Caractérisation de la végétation ligneuse et des organisations pelliculaires de surface des agroécosystèmes à différents stades de dégradation de la Commune rurale de Simiri (Niger) Int. J. Biol. Chem. Sci., 7(5): 1963-1975. DOI : http://dx.doi.org/10.4314/ijbcs.v7i5.15.

Dan Guimbo I. 2011. Fonction, dynamique et productivité des parcs à Vitellaria paradoxa C.F. Gaertn. et à Neocarya macrophylla (Sabine) Prance dans le sud-ouest du Niger. Université Abdou Moumouni de Niamey, Thèse de Doct. Sces Agro. et Ing. Géog., 191p.

Douma S. 2009. Ecologies de quatre espèces ligneuses de la reserve totale de faune de Tamou: Indicateurs de gestion durable des ressources forestières, Mémoire de DEA de Biologie, Université Abdou Moumouni de Niamey, p.73.

Douma S, Chaibou R, Mahamane A, N'DA DH, Saadou M. 2010. Etat actuel de dégradation des populations de quatre espèces ligneuses fruitières en zone sahélo-soudanienne du Niger : Réserve totale de faune de Tamou. Rev. Ivoir. Sci. Technol., 16: 191 - 210.

Dufrêne M, Legendre P. 1997. Species assemblages and indicators species: the need for a flexible asymmetrical approach. Ecological Monographs, 67: 345-366.

FAO. 2010. Evaluation des ressources forestières mondiales. Rapport principal, Etude FAO, Forêts Rome, Italie, 163: p.34.

Ganaba S. 2008. Caractérisation, utilisations, tests de restauration et gestion de la végétation ligneuse au Sahel, Burkina Faso. Thèse de Doctorat d'Etat, Université Cheikh Anta Diop, p.287.

Ichaou A. 2000. Dynamique et productivité des structures forestières contractées des plateaux de l'ouest Nigérien. Thèse, Université Paul Sabatier de Toulouse III, p.231.

Larwanou M, Saadou M. 2011. The role of human interventions in tree dynamics and environmental rehabilitation in the Sahel zone of Niger. Journal of Arid Environments, 75(2): 194-200.

Mahamane A, Saadou M. 2008. Méthode d'étude de la flore et de la végétation tropicale. Project SUN-EU. Actes de 
l'Atelier sur l'Harmonisation des Méthodes, p.83.

Matsallabi A. 2008. Ressources ligneuses et problématique d'aménagement dans la forêt classée de Dan Kada-Dodo (Département d'Aguié). Rapport de stage pour l'obtention du diplôme de Maîtrise ès-sciences Agronomiques, Université Abdou Moumouni de Niamey, p.61.

Mbayngone E, Thiombiano A, Hahn-Hadjali K, Guinko S. 2008. Structure des ligneux des formations végétales de la Réserve de Pama (Sud-Est du Burkina Faso, Afrique de l'Ouest). Flora et Vegetatio Sudano-Sambesica, 11: 25-34.

McCune B, Grace JB. 2002. Analysis of ecological communities. Gleneden Beach, Oregon: MJM Software Design, p.300.

Morou B. 2010. Impacts de l'occupation des sols sur l'habitat de la girafe au Niger et enjeux pour la sauvegarde du dernier troupeau de girafes de l'Afrique de l'Ouest. Thèse de Doctorat unique, Université Abdou Moumouni de Niamey, Niger, p.198.

Ndong AT, Ndiaye O, Sagna M.B, Diallo A, Galop D, Guisse A. 2015. Caractérisation de la végétation ligneuse sahélienne du Sénégal: cas du Ferlo. Int. J. Biol. Chem. Sci., 9(6): 2582-2594.
DOI:

http://dx.doi.org/10.4314/ijbcs.v9i6.6.

Ouédraogo A. 2006. Diversité et dynamique de la végétation ligneuse de la partie orientale du Burkina Faso. Thèse de doctorat, Univ. Ouagadougou, p.196.

Ouédraogo O. 2009. Phytosociologie, dynamique et productivité de la végétation du parc national d'Arly (SudEst du Burkina Faso). Thèse de doctorat, Université de Ouagadougou, p.188.

Ouôba P. 2006. Flore et végétation de la forêt classée de Niangoloko, Sud-Ouest du Burkina Faso. Thèse de Doctorat, Université de Ouagadougou, p.144.

Paré S. 2008. Land Use Dynamics, Tree Diversity and Local Perception of Dry Forest Decline in Southern Burkina Faso, West Africa. Doctoral Thesis, Swedish University of Agricultural Sciences, p.78.

Rondeux J. 1999. La Mesure des Peuplements Forestiers. Presses Agronomiques: Gembloux ; 522.

Sambou B. 2004. Evaluation de l'état, de la dynamique et des tendances évolutives de la flore et de la végétation ligneuses dans les domaines soudanien et subguinéen au Sénégal. Thèse de doctorat, Université Cheikh Anta Diop, p. 248 . 